\title{
Reconstrucción de ligamento colateral medial: descripción de una nueva técnica de aumentación con aloinjerto y tornillos biocompuestos interferenciales
}

\author{
Reconstruction of medial collateral ligament: Description of a new augmentation \\ technique with allograft and interference biocomposite screws
}

Parroquín-Maldonado JA, * Mas-Celis F, ${ }^{\ddagger}$ Cruz-Miranda A ${ }^{\S}$

Hospital Ángeles Lomas, México.

RESUMEN. Introducción: La inestabilidad del ligamento colateral medial (LCM) se asocia con lesiones multiligamentarias. Existen varios procedimientos para la reconstrucción del LCM, presentamos una técnica percutánea de aumentación. Nuestro objetivo es describir una nueva técnica de reconstrucción del LCM mediante injerto y fijación con tornillos biocompuestos. Material y métodos: Presentamos la técnica en un total de 21 pacientes consecutivos con lesión del LCM operados en el período de Diciembre de 2011 a Octubre de 2014. La reconstrucción del LCM se realizó con aloinjertos del tendón del peroneo largo, tibial posterior o flexor largo del Hallux en 18 pacientes y solamente en un paciente se utilizó autoinjerto. De los 20 pacientes, 18 presentaron lesiones asociadas: cinco con lesión de menisco medial, ocho con lesión del ligamento cruzado anterior, (LCA), ocho con lesión condral y uno con lesión del menisco lateral. Conclusión: La técnica quirúrgica presentada es sencilla de realizar, sin daño a otras estructuras y con una fijación resistente.

Palabas clave: Inestabilidad medial, ligamento colateral medial, reconstrucción anatómica, radiografías en estrés, rodilla, aloinjertos.

\section{Nivel de evidencia: IV}

* Ortopedista adscrito del Hospital Ángeles Lomas.

‡ Ortopedista adscrito del Hospital Ángeles Pedregal.

${ }^{\S}$ Ortopedista adscrito del Centro Médico ABC.

Dirección para correspondencia:

Dr. Javier Alberto Parroquín-Maldonado

Col. Valle de las Palmas, C.P. 52763, Hacienda de las

Palmas, Huixquilucan,

Méx. Tel: 52469520

E-mail: yevier@aol.com

https://dx.doi.org/10.35366/95328

doi: $10.35366 / 95328$
ABSTRACT. Introduction: Medial Collateral Ligament (LCM) instability is associated with multiligamentary lesions. There are several procedures for the reconstruction of MCL, we present a percutaneous technique of augmentation. Our goal is to describe a new technique of reconstruction of the LCM by grafting and fixing with biocomposite screws. Material and methods: We present the technique in a total of 21 consecutive patients with MCL injury operated in the period of December 2011 to October 2014. Reconstruction of MCL was performed with long, tibial or long hallux tendon allografts in 18 patients and only one patient was used autograft. Eighteen of the 20 patients had associated lesions: 5 with medial meniscus injury, 8 with anterior cruciate ligament injury, (ACL), 8 with condral injury and 1 with lateral meniscus injury. Conclusion: The surgical technique presented is simple to perform, without damage to other structures and with a strong fixation.

Keywords: Medial instability, medial collateral ligament, anatomical reconstruction, stress x-rays, knee, allografts.

\section{Introducción}

El ligamento colateral medial (LCM) es una de las estructuras de la rodilla que más comúnmente se lesiona y representa hasta $42 \%$ de todas las lesiones ligamentosas de rodilla. Sabemos que como consecuencia de la deficiencia de algún ligamento, el estrés de contacto tibiofemoral anormal puede llevar a la progresión de la osteoartritis. ${ }^{1}$ La mayoría de los casos (grado I Hughston o II) son tratados en forma conservadora debido a la alta capacidad de reparación endógena. Sin embargo, en situaciones en las que la laxitud en valgo es severa (Hughston grado III), se presenta con lesiones asociadas de rodilla o en casos crónicos, es preferible optar por el tratamiento quirúrgico. ${ }^{2}$ 
El ligamento colateral medial superficial (LCMs) forma parte de los estabilizadores estáticos de la parte medial de la rodilla. Tiene un aspecto acintado y posee un único punto de inserción femoral y dos inserciones tibiales distales. La inserción femoral se localiza a $3.2 \mathrm{~mm}$ proximal y a $4.8 \mathrm{~mm}$ posterior al epicóndilo medial. En su trayecto distal tiene una primera inserción tibial proximal a partes blandas en íntima relación con el tendón reflejo del semimembranoso aproximadamente a $12.2 \mathrm{~mm}$ distal a la interlínea articular. El sitio de inserción distal del LCMs es extenso y en promedio $61.2 \mathrm{~mm}$ distal a la interlínea articular, localizado inmediatamente anterior a la cresta posteromedial de la tibia y se encuentra en íntima relación a la bursa de los tendones de la pata de ganso. Estos dos sitios distales son importantes referencias, ya que se les han atribuido propiedades biomecánicas diferentes. El principal estabilizador en valgo durante todos los grados de flexión de rodilla es el LCMs en su inserción tibial proximal, mientras que la inserción distal se encarga fundamentalmente de la estabilidad en rotación externa a 30 y $60^{\circ}$ de flexión de rodilla y la rotación interna (Figura 1).

Sin olvidar que el estándar de oro para el diagnóstico de una lesión LCM es por medio de una exploración clínica, se pueden utilizar estudios de gabinete como herramientas auxiliares. Las radiografías deberían adoptarse para descartar cualquier fractura. Las radiografías en estrés son la técnica más precisa y reproducible disponible actualmente; sin embargo, sólo un número limitado de estudios han utilizado este método para documentar sus resultados posteriores a la reconstrucción del LCM. Una resonancia magnética puede ser útil para visualizar los ligamentos y otros tejidos blan-
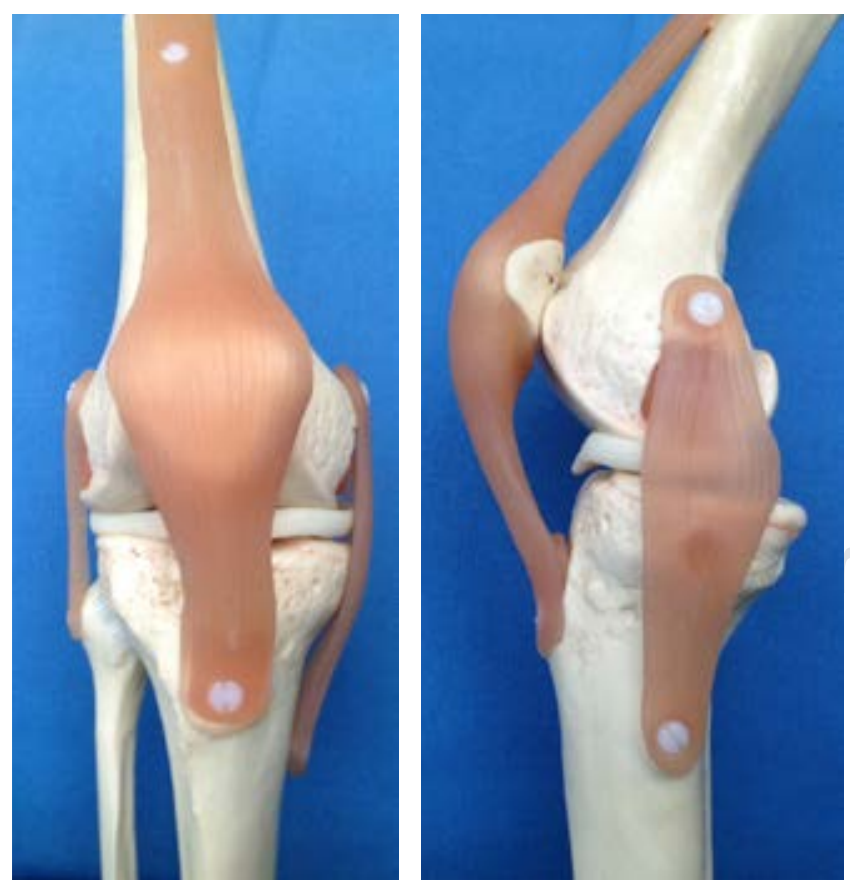

Figura 1: Representación del LCMs como estabilizador en valgo durante la flexión. dos. ${ }^{2,3}$ Los principios de tratamiento quirúrgico incluyen técnicas de reparación y reconstrucción. En la literatura las técnicas de reconstrucción sólo han abordado la porción LCMs o también se ha descrito en combinación con las estructuras posteromediales como el ligamento oblicuo posterior (POL) o la cápsula posteromedial. Algunos autores han descrito la reconstrucción del LCM mediante autoinjertos o aloinjertos de tendón del semimembranoso o del Aquiles. ${ }^{4,5}$

Decidimos utilizar tornillos biocompuestos en la técnica propuesta, ${ }^{6,7}$ ya que el avance en los nuevos polímeros y materiales promueven la osteointegración a través de la fijación biológica. Los tornillos de interferencia biocompuestos son reemplazados por nuevo hueso en 36 meses in vivo. Está comprobado que este tipo de tornillos proporciona estabilidad a largo plazo con injertos para reconstrucción de ligamento cruzado anterior al permitir la transmisión efectiva de las fuerzas de carga.

La reconstrucción del LCM se ha asociado a complicaciones relacionadas a una amplia exposición de tejidos, riesgo de morbilidad del sitio donante con autoinjertos, pérdida de movimiento o la colocación no anatómica del injerto.

El presente trabajo tiene como objetivo describir una nueva técnica de reconstrucción del LCM mediante injerto y fijación con tornillos biocompuestos.

\section{Material y métodos}

Los criterios de inclusión fueron: (1) inestabilidad de rodilla con las actividades de la vida cotidiana y/o con algunas de mayor esfuerzo como actividades deportivas, (2) hombres y mujeres mayores de 15 años de edad, (3) lesión primaria del LCM, (4) inestabilidad medial comprobable clínicamente con un bostezo aumentado en flexión de $20^{\circ} \mathrm{y}$ en extensión completa comparado con la rodilla contraria.

Las complicaciones potenciales incluyeron:

Sección del nervio poplíteo externo.

Sangrado.

Rotura o desprendimiento del injerto durante rehabilitación extrema.

Todos los pacientes fueron sometidos a reconstrucción de LCM durante el período comprendido entre Diciembre de 2011 y Marzo de 2015 por un mismo cirujano; 16 pacientes dentro de nuestra institución y tres casos en otras instituciones. Para evaluar el grado de laxitud durante la exploración física se realizaron maniobras de inestabilidad en valgo forzado.

En la Tabla 1 se resumen los datos demográficos de la muestra de pacientes.

\section{Técnica quirrúrgica}

Con el paciente bajo efectos de anestesia general balanceada y en posición decúbito supino, se coloca torniquete neumático en muslo de extremidad a operar programado a 
Tabla 1: Características demográficas del grupo de estudio.

\begin{tabular}{|c|c|c|c|c|c|}
\hline Paciente & Edad & Género & Lateralidad & Mecanismo de lesión & Lesiones asociadas \\
\hline 1 & 32 & Masculino & Derecha & Caída de escalera & MM \\
\hline 2 & 38 & Masculino & Izquierda & Accidente automovilístico & LCA \\
\hline 3 & 40 & Masculino & Izquierda & Actividad deportiva & LCA, Desprendimiento polo superior patella \\
\hline 4 & 47 & Femenino & Izquierda & Actividad deportiva & LCA, MM \\
\hline 5 & 28 & Masculino & Izquierda & Caída & Revisión LCA \\
\hline 6 & 45 & Femenino & Izquierda & Caída & Lesión condral, MM \\
\hline 7 & 50 & Femenino & Izquierda & Actividad deportiva & Lesión Bankart, Lesión condral \\
\hline 8 & 60 & Femenino & Derecha & Artrosis degenerativa & Lesión condral, Artrosis degenerativa \\
\hline 9 & 60 & Femenino & & Inestabilidad postraumática & Lesión condral \\
\hline 10 & 33 & Femenino & Izquierda & Caída & Lesión condral \\
\hline 11 & 38 & Femenino & Izquierda & Rehabilitación insuficiente & Revisión LCA \\
\hline 12 & 39 & Masculino & Derecha & Accidente automovilístico & Lesión condral \\
\hline 13 & 35 & Femenino & Izquierda & Caída postquirúrgica (7 meses) & \\
\hline 14 & 16 & Femenino & Izquierda & Actividad deportiva & LCA, Menisco discoide \\
\hline 15 & 25 & Femenino & Derecha & Actividad deportiva & LCA (autodonador), MM (autodonador) \\
\hline 16 & 16 & Femenino & Derecha & Actividad deportiva & Lesión LCM aislado \\
\hline 17 & 35 & Femenino & Derecha & Caída de escalera & LCA, MM \\
\hline 18 & 43 & Femenino & Derecha & Caída de escalera & Lesión condral, ML \\
\hline 19 & 19 & Masculino & Derecha & Actividad deportiva & Lesión condral \\
\hline
\end{tabular}

$300 \mathrm{mmHg}$. En todos los pacientes se realizó asepsia y antisepsia de la extremidad con Isodine espuma y ChloraPrep ${ }^{\circledR}$. Se llevó a cabo una artroscopía de rodilla mediante técnica de tres portales con revisión sistemática de la articulación. Se describieron las lesiones observadas y se hizo reparación en caso necesario. Se termina artroscopía y se continúa con la aumentación percutánea del LCM.

Se identifica la posición del ligamento colateral medial anatómico (Figura 2), localizado en el vértice superior del epicóndilo medial del fémur y se realiza incisión de $1 \mathrm{~cm}$ de longitud con disección roma hasta identificar cortical ósea. Se introduce guía metálica de forma perpendicular al eje femoral hacia el lado opuesto.

Se elabora un túnel del ancho del aloinjerto $(7 \mathrm{~mm})$ y se introduce guía con orificio para el paso del aloinjerto (tendón peroneo largo, flexor largo del hallux o tibial posterior) en dirección de medial a lateral y se extrae sutura en borde lateral del fémur. Mediante sistema de biotenodesis se fija aloinjerto con un tornillo (BioComposite Interference Screw ${ }^{\circledR}$ Arthrex) (Figura 3).

Posteriormente se hace incisión de $1 \mathrm{~cm}$ de longitud 10 $\mathrm{mm}$ distales a la superficie articular del platillo tibial medial. Mediante fluoroscopía se identifica zona y de igual forma se realiza túnel, teniendo en cuenta la anatomía para evitar lesionar el nervio peroneo común. Se introduce guía de nitinol con orificio para el otro cabo de sutura del aloinjerto. Se aplica tensión al aloinjerto desde la sutura en superficie la- teral de la rodilla para posteriormente fijarlo con un tornillo biocompuesto (BioComposite Interference Screw ${ }^{\circledR}$ Arthrex) en la región medial, simultáneamente se realizan maniobras en varo de $10-15^{\circ}$ (Figura 4). Se hacen pruebas de estabilidad clínica y radiografías de estrés. Se cortan hilos de sutura

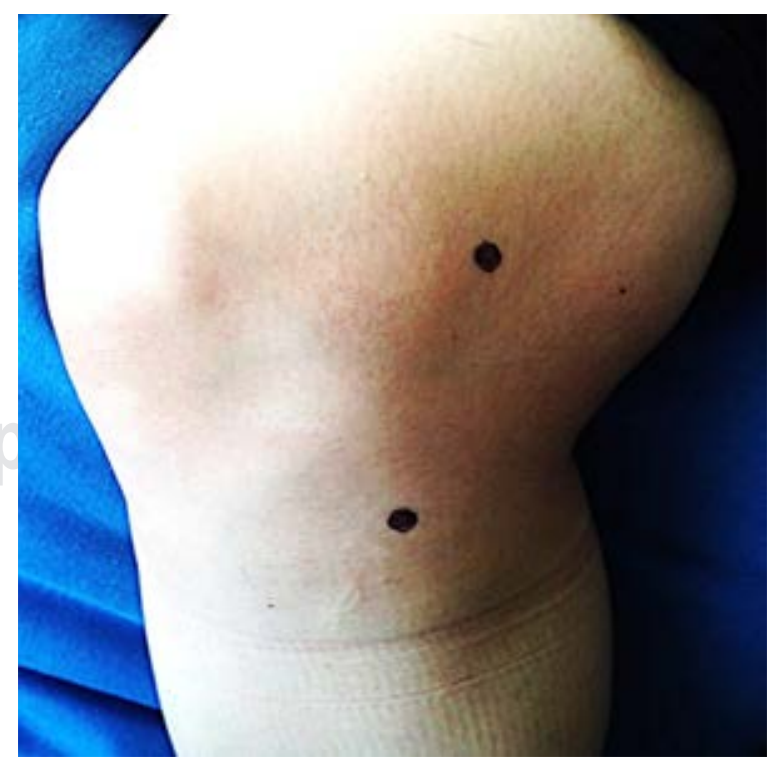

Figura 2: Identificación del sitio anatómico del sitio de origen del ligamento colateral medial. 
en superficie lateral de la rodilla a nivel de la piel. Se suturan heridas con Vicryl y Dermalón. Se cubren y se coloca rodillera articulada a libre movimiento.

\section{Postquirúrgico}

Una vez terminado el procedimiento, las heridas se cubren con gasas y se coloca una rodillera articulada con ángulo libre de flexibilidad. Se retiran puntos de sutura a los 15 días postoperatorios. Realizamos seguimiento cada 10 días para vigilar la presencia de dolor, inestabilidad residual o secundaria y cuidar el rango de movimiento. Por seis semanas se permiten arcos de movimiento completos activos y pasivos, ejercicios isométricos e isotónicos así como fortalecimiento muscular sin gravedad. Es de suma impor- tancia que los ejercicios se realicen de forma simétrica a la articulación contralateral.

Posteriormente se cita cada mes por los siguientes seis meses con una radiografía de control. Se inician actividades contra gravedad y resistencia, pero de bajo impacto (caminatas largas, trote).

Los deportes de alto rendimiento se permiten a los seis meses postoperatorios una vez finalizados los ejercicios de propiocepción.

\section{Discusión}

La restauración anatómica del LCM con nuestro procedimiento quirúrgico funciona, esta técnica de reconstrucción percutánea es un procedimiento útil y se consigue la estabi-
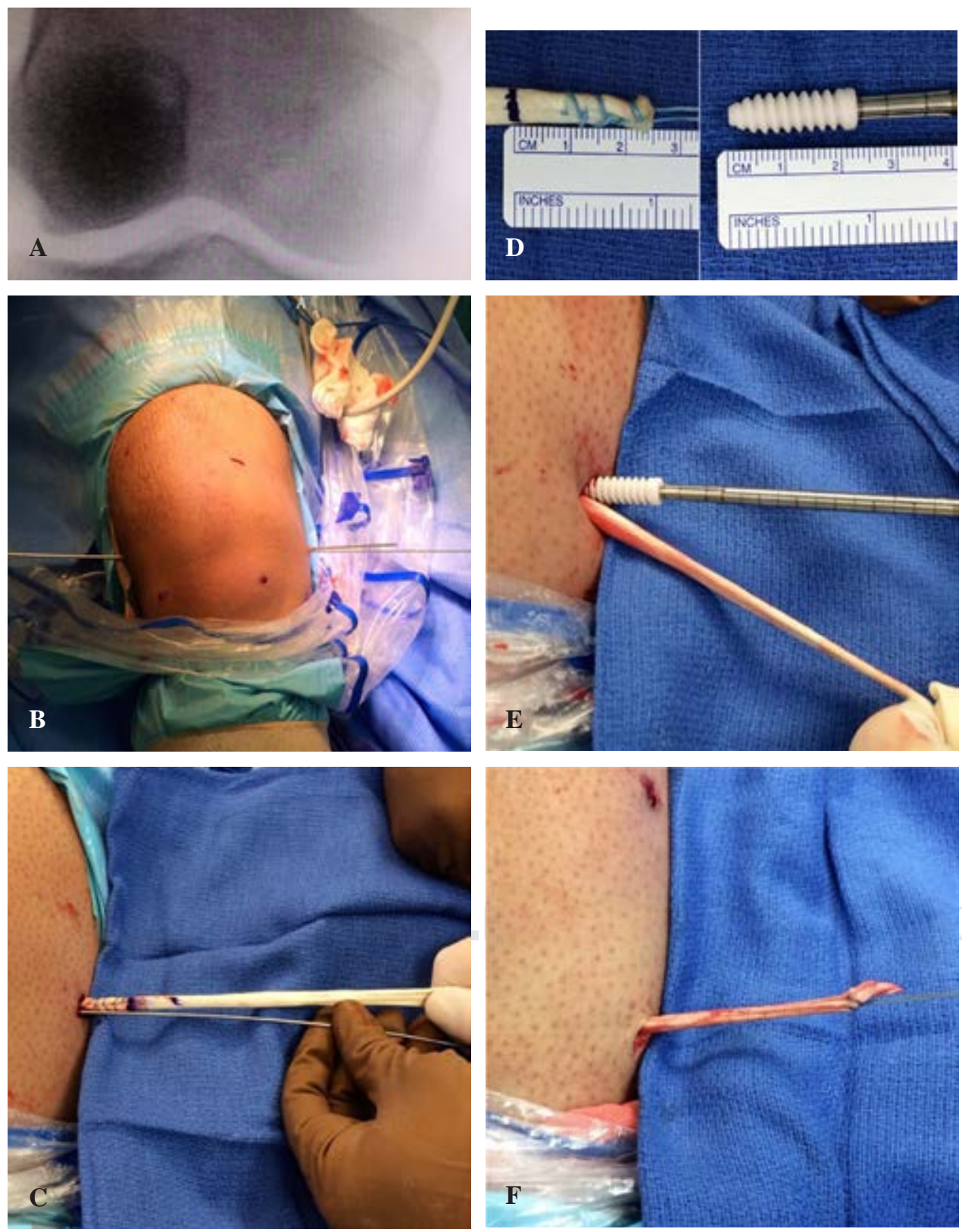
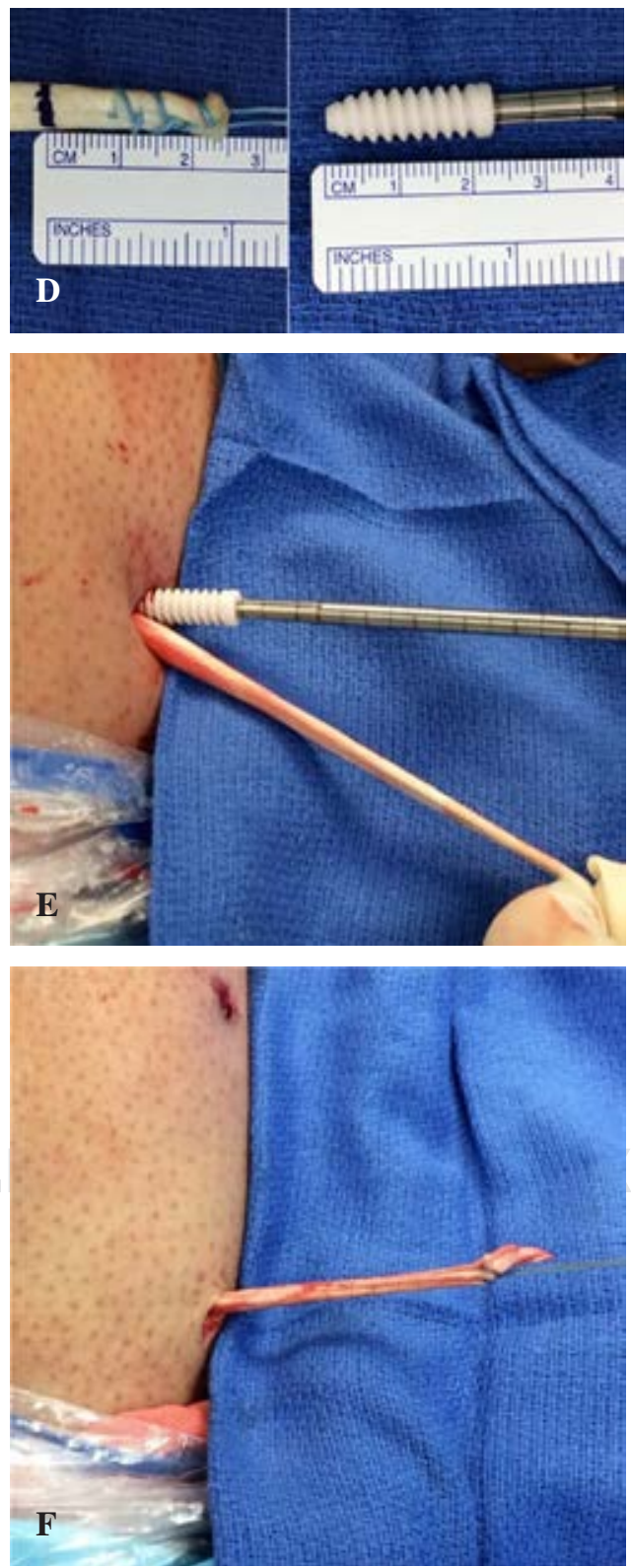

(1)

1
Figura 3:

A) Identificación por fluoroscopía del vértice superior del epicóndilo medial. B) Introducción de guía metálica hacia el lado opuesto. C) Paso de injerto de medial a lateral. D) Medición del cabo femoral del injerto. E) Fijación. F) Paso de injerto de forma subcutánea hacia la incisión tibial. 

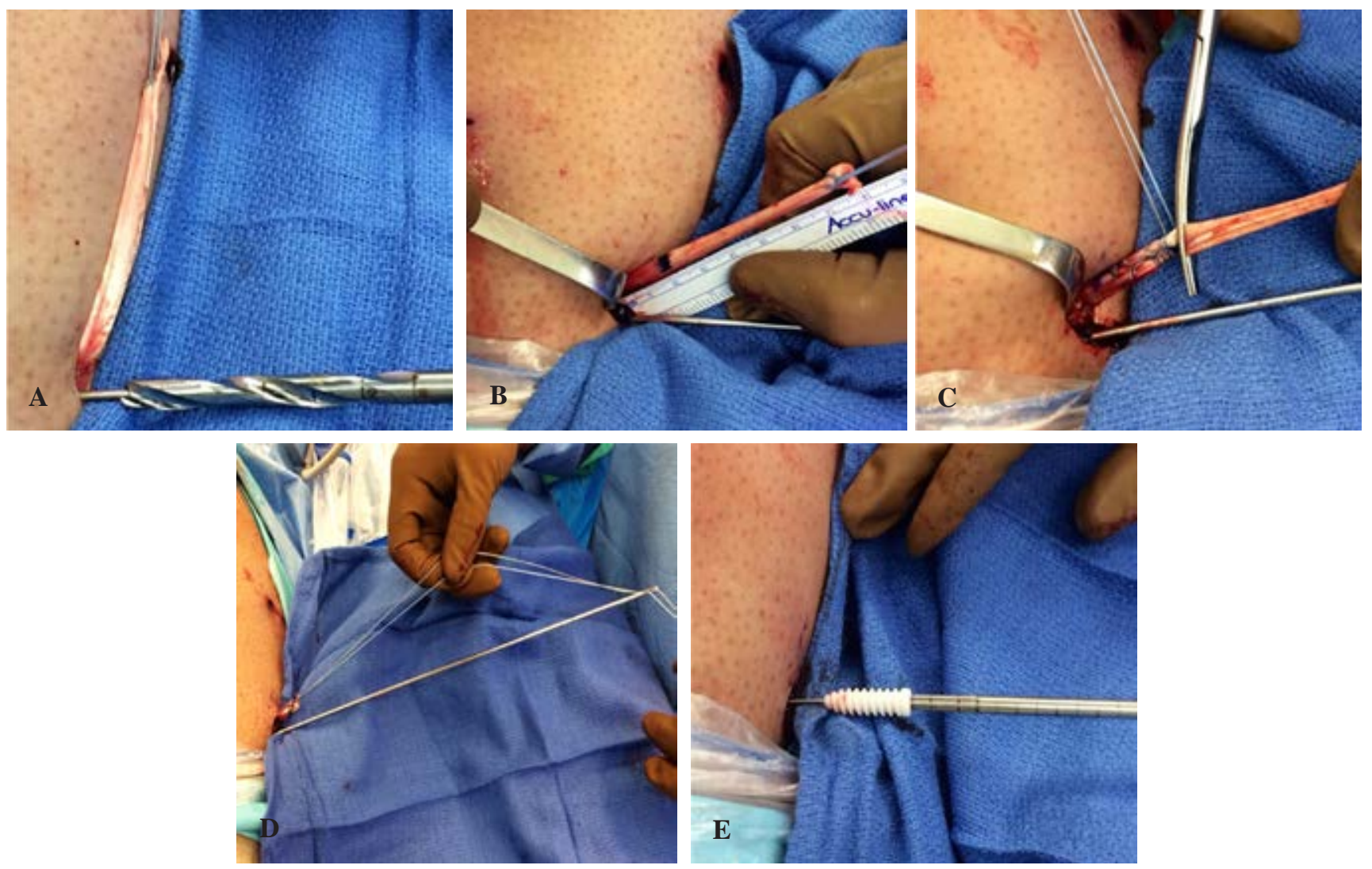

Figura 4: A) Elaboración del túnel tibial. B) Medición del cabo distal. C) Recorte del extremo terminal. D) Paso de injerto por el túnel tibial. E) fijación con tornillo aplicando al mismo tiempo maniobra de Varo.

lidad de la rodilla con uso de un aloinjerto. Es una cirugía conveniente, rápida y eficaz con resultados favorables a corto plazo y comparable a técnicas ya descritas en la literatura.

Para enriquecer nuestro estudio, se pretende extender la revisión de los casos para dar a conocer la biointegración de los tornillos interferenciales de los injertos con el tejido óseo.

Hangzhou Zhang y colaboradores describieron la técnica de fijación tibial para la reconstrucción de LCM utilizando un aloinjerto de Aquiles (hueso-tendón-hueso), que reproduce la anatomía y la orientación del MCL. Sin embargo, su técnica es una cirugía abierta con incisión más grande. La presentación de una técnica percutánea como la que hemos descrito $^{8}$ no presenta reportes similares.

Bibliografía

1. Omar M, Petri M, Dratzidis A, Nehmer SE, Hurschler C, Krettek C, et al. Biomechanical comparison of fixation techniques for medial collateral ligament anatomical augmented repair. Knee Surg Sport Traumatol Arthrosc. 2016; 24(12): 3982-7.
2. Liu X, Feng H, Zhang H, Hong L, Song WX, Zhang J, Wei SJ. Surgical treatment of subacute and chronic valgus instability in multiligament-injured knees with superficial medial collateral ligament reconstruction using Achilles allografts: a quantitative analysis with a minimum 2-year follow-up. Am J Sports Med. 2013; 41: 1044-50.

3. Marx RG, Hetsroni I. Surgical technique: medial collateral ligament reconstruction using Achilles allograft for combined knee ligament injury. Clin Orthop Relat Res. 2012; 470: 798-805.

4. LaPrade RF, Engebretsen AH, Ly TV, Johansen S, Wentorf FA, Engebretsen L. The anatomy of the medial part of the knee. $J$ Bone Joint Surg Am. 2007; 89: 2000-10.

5. Griffith CJ, LaPrade RF, Johansen S, Armitage B, Wijdicks C, Engebretsen L. Medial knee injury. Part 1. Static function of the individual components of the main medial knee structures. Am J Sports Med. 2009; 37: 1762-70.

6. Jensen, et al. Journal of biomedical materials research part B. Appl Biomater. 2009; 90B: 171-81.

7. Suchenski M, McCarthy MB, Chowaniec D, Hansen D, McKinnon W, Apostolakos J, et al. Material properties and composition of soft-tissue fixation. Arthroscopy. 2010; 26(6): 821-35.

8. Zhang H, Bai X, Sun Y, Han X. Tibial inlay reconstruction of the medial collateral ligament using Achilles tendon allograft for the treatment of medial instability of the knee. Knee Surg Sports Traumatol Arthrosc. 2014; 22: 279-84. 